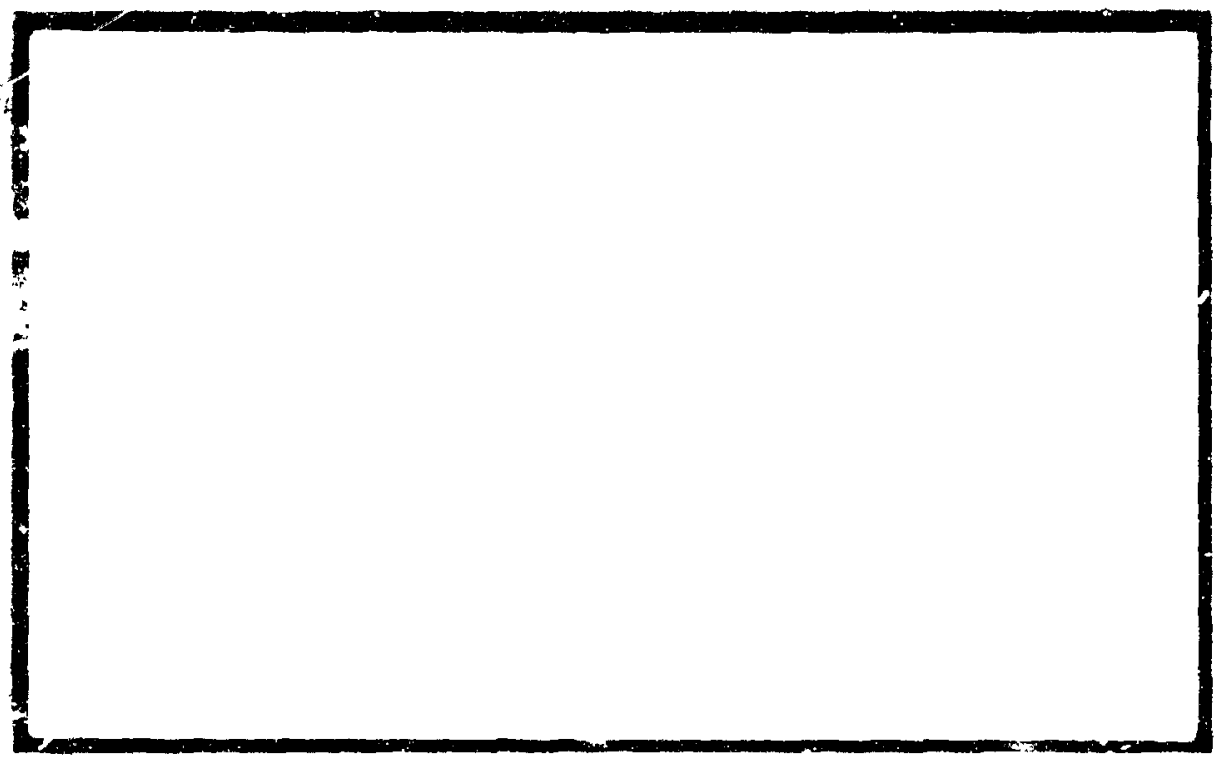

i

(AASA-CR-162524) A. ALYSISCF EESL SXSEE

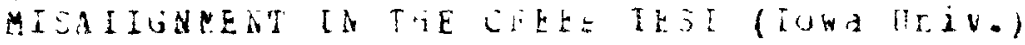

$10 u-2.2+06$

43 P HC AO? RT AU1

Division of Moterials Engineering

COLLEGE OF ENGINEERING

THE UNIVERSITY OF IOWA

Iowa City lowa 52242

$\bullet$

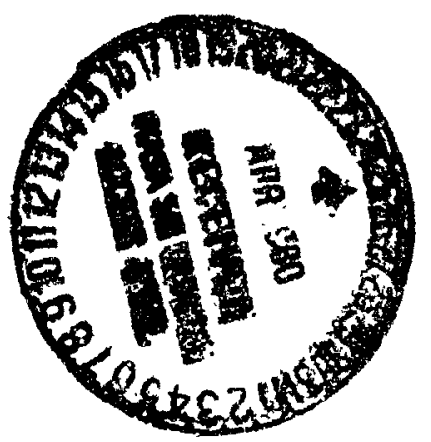




\section{ANALYSIS OF TEST SYSTEM MISALIGNMENT \\ IN THE CREEP TEST}

by

Han C. Wu and T. P. Wang

Division of Materials Englneering

The University of Jowa

Iowa city, Iowa 52242

Report (3302-80-002

Propared for

NASA-langley Research Center

Hampton, Virginia

Grant No. NSG 1499

April 15, 1980 


\section{Abstract}

An analysis of test system misalignment is presented for the creep test. Sheet type rectangular 1100-0 aluminum specimens are used for discussion. It is found that the creep strain at the geometric centerline of the specimen is different than that at the neutral axis. However, this difference in the creep strain decreases with time. Generally, the effect of misalignment decreases with creep time.

Creep tests conducted with long pullrods and large inftial strain level (high creep stress) will tend to minimize the effect of misalignment. 


\section{INTRODUCTION}

The effect of misalignment in a tensile test system has been previously Investigated by a number of investigators. These Investigators have also Identifled the sources of misalignment as poor conformance of specimen centerline to top and bottom grip centerlines, poor alignment of the top and bottom grip centerlines, and inaccurate machining of the test specimen 1tself. A more detalled description of test system misalignment and the discussion concerning the need for such investigation may be found in Penny and Leckie [1], Christ and Swanson [2], and Wu and Rummler [3].

The misalignment in the creep test was previously investigated by Penny and Leckie [1], Hayhurst [4], and Penny et al. [5]. These research workers generally agreed that data scatter in creep testing could be partially attributed to specimen alignment. Poor specimen alignment could result in bending stresses (or strains) superimposed on the required mean axial stress (or strain). This bending stress (or strain) could be high enough to cause premature fallure in brittle materials; in ductile materials, pjastic deformation could occur and reduce the bending stresses (or strains).

Analyses of misalignment were presented in Ref. [1 and 4] for the creep test. The results show that the creep curves "scatter" due to eccentric loading. More significantly, these analyses predicted that the "scatter" would increase with the creep time.

The result of the present analysis does not support all of the above assertions. While the numerical results show that the errors induced by the test system misalignment are significant in the creep test, these errors will diminish with time. Thus, the most severe effect of misalignment occurs at the beginning of the creep test. 
The analysis is performed on rectangular specimena of the sheet type. Specimens of 1100-0 aluminum ldve been chosen for investigation. The reason for using this material as an example for discussion is that the constitutive equation employed in the present analysis has been shown to describe both the dynamic stress-strain behavior and creep for this material [6] and the material constants have already been determined. Theoretically speaking, the method presented herein would also be applicable to other materials.

Only the symmetric case of misalignment is studied due to the finding of Ref. [3] that this is the most severe case of all the cases investigated. The endochronic constitutive equations developed by Wu and Chen [6] for creep are used in the analysis. However, it is believed that the results obtained in this investigation concerning the effect of misalignment is independent of the constitutive model used. Any consitutive creep equation which can correctly describe the creep behavior may be used for the misalignment analysis.

The present analysis is the third part of a series of investigation ralated to test system misalignment. The first part was presented in Wu and Rummler [3] which reported a misalignment analysis for the tension test under static loading condition. The second part was given in wu et al. [7] and reported an analysis of the tension test under dynamic loading condition. In particular, the effect of strain-rate was investigated in Ref. [7]. The results of this work also serve as the inftial input to the present calculation. 


\section{CONSTITUTIVE EQUATION FOR CREEP}

The present analysis utilizes a constitutive equf, $\{$ jon which was shown by $\mathrm{Wu}$ and Chen [6] to describe the transient creep and creep recovery. In this approach, the deformation history of the specimen prior to the onset of creep is important and significantly affects the transient creep be'lavior. Therefore, the whole creep test is viewed to have two stages. The first stage is the loading stage and the second stage is the creep stage (see Fig. 1).

The loading stage is treated as a constant strain-rate tension test (see Ref. [6] for details). The numerical results reported in Ref. [7] are precisely the numerical data for this stage of the test. The creep stage is described by the following equations (see Ref. [6]):

$$
\begin{aligned}
k_{c}(\dot{\theta})= & \sigma_{y}^{o}(1+\beta z) /\left\{\sigma *-R(z)-\frac{\sigma_{0}^{0}-\sigma_{y}^{0}}{k_{0}}(1+\beta z) .\right. \\
& {\left.\left[\left(\frac{1+\beta z_{0}}{1+\beta z_{0}}\right)^{n}-\left(1+\beta z_{z}\right)^{-n}\right]\right\} }
\end{aligned}
$$

and

$$
d z_{c}=k_{c}(\dot{\theta})|a \theta|
$$

where

$$
k_{c}(\dot{\theta})=1-\beta_{c} \ln \left(\frac{\dot{\theta}}{\dot{\theta}_{R}}\right)
$$




$$
\begin{aligned}
R^{(}\left(z_{0}\right)= & -\left(1+\beta z_{2}\right)^{-A / \beta}\left\{\frac{C_{1}}{A-\alpha}\left[\left(1+\beta z_{1}\right)^{(A-\alpha) / \beta}-\left(1+\beta z_{0}\right)^{(A-\alpha) / \beta}\right]\right. \\
& \left.-\frac{C_{2}}{A}\left[\left(1+\beta z_{2}\right)^{A / \beta}-\left(1+\beta z_{0}\right)^{A / \beta}\right]\right\}
\end{aligned}
$$

$A=\frac{E_{1}}{\sigma_{y}^{0}}+\alpha$

$c_{1}=\frac{E_{1}}{\sigma_{y}^{0}} \frac{\sigma_{0}^{0}-\sigma_{y}^{o}}{k_{0}}\left[\left(1+\beta z_{0}\right)^{n}-1\right]$

$$
C_{2}=\frac{E_{1} \sigma *}{\sigma_{y}^{\circ}}
$$

$$
E_{1}=\beta k_{0} n\left(\sigma_{0}^{0}-\sigma_{y}^{0}\right)
$$

and

$$
\alpha=B(n-1)
$$

In the above equations, $\sigma_{y}^{\circ}, \sigma_{0}^{\circ}, \beta, n$ and $\dot{\theta}_{R}$ are material constants determined by a set of constant strain-rate stress-strain curves; $\sigma^{*}$ 1s the cresp stress which is a constant; $\theta$ is the creep strain; $k_{0}$ and $z_{0}$ are values determined at the end of the loading stage; and $\beta_{c}$ depends on $\sigma *$ as a parameter.

Rearranging equation (3), the creep rate is expressed by

$$
\dot{\theta}=\dot{\theta}_{R} \exp \left\{\left[1-k_{c}(\dot{\theta})\right] / \beta_{c}\right\}
$$


Thus, equations (1), (2) and (10) form the basic equations describing the transient creep.

To compute the creep curve, a step-by-step numerical scheme should be employed. Generaliy, the current value of $k_{c}(\dot{\theta})$ ts computed from equation (1) by use of the value of $z$ at the previous time step. The current value of $k_{c}(\dot{\theta})$ is then kept constant when the increments of the creep strain and the value of $z$ are being computed from equations (10) and (2). 


\section{ANALYSIS OF MISALIGMMENT}

As mentioned in the previous section, the creep test under investigation is a constant strain-rate loading test followed by a constant stress creep test. In the loading stage the strain-rate is constant at the neutral axis (N.A.) of the specimen. In the creep stage, the stress along the N.A. remains constant, but it changes with time at other parts of the specimen due to the effect of misalignment.

In fact, phenomena such as creep recovery and stress relaxation may indead occur at the part of cross-section outside of the N.A. However, the stress variation for a given fiber at each time step of computation is so small that the effect of creep recovery may be ignored compared with the creep strain resulted from the constant stress. This assumption w1ll be justified by the numerical results.

The assumption of plane cross-sections remaining plane during deformation is made in the analysis. The coordinate system is same as that used In Ref. [3 and 7] and shown in Fig. 2a. The essential features of the symmetric case of misalignment is also presented in Fig. 2b. The discretized numerical model is identical to that of Ref. [7] and is reproduced in Fig. 3 . Due to symmetry, only half of the specimen is considered, which is then divided into $I$ and $J$ sections along the longitudinal and the lateral direction, respectively.

At a particular time $t$ in cree?, the planes remaining plane assumption requires that

$$
\varepsilon^{t}(x, y)=e^{-t}-\frac{y^{t}}{\rho^{t}(x)}
$$




$$
\rho^{t}(x)=\frac{h}{\varepsilon_{B}^{t}-\varepsilon_{T}^{t}}
$$

and

$$
d^{t}(x)=h \frac{\varepsilon_{B}^{t}-\varepsilon^{-t}}{\varepsilon_{B}^{t}-\varepsilon_{T}^{t}}
$$

where $x$ and $y$ are coordinates sinown in Fig. $2(a) ; \bar{\varepsilon}$ is the strain on the N.A.,; $\rho(x)$ is the radius of curvature of the specimen; $\varepsilon_{T}$ and $\varepsilon_{B}$ are the top and bottom ftbers, respectively; $h$ is the width of the specimen; and $d(x)$ specifies the location of the N.A, and is measured from the bottom fiber. The superscript $t$ indicates that the quantities are referred to the time step $t$.

The procedure for solution is such that the quantities at the N.A., represented by the barred quantities, should be assigned first. This may be done by use of equations (1), (2), and (10). Therefore,

$$
\begin{aligned}
& \dot{\bar{\theta}} t=\dot{\theta}_{K} \exp \left\{\left(1-\bar{k}_{c}^{t-\Delta t}\right) / \bar{\beta}_{c}\right\} \\
& \bar{\varepsilon} t=\bar{\varepsilon}^{-t-\Delta t}+\dot{\bar{\theta}}^{t} \Delta t \\
& \bar{z}^{t}=\bar{z}^{t-\Delta t}+\bar{k}_{c}^{t-\Delta t} \cdot \dot{\bar{\theta}}^{t} \cdot \Delta t
\end{aligned}
$$

The creep behavior at the N.A. is same as that of a perfectly aligned specimen. Quantities at other fibers are then determined by means of the misalignment analysis given in the remaining part of this section. 
At a given time $t$, balance of load is

$$
P=\int_{A} \sigma^{t} d A=\frac{b h}{J} \sum_{j=1}^{J} \sigma_{1 j}^{t} ; 1=1, \ldots, I+1
$$

in which $P$ is the force, $b$ is the thickness, A is the cross-sectional area, and $h / J$ is the width of the element under consideration at the $1^{\text {th }}$ section. The balance of moment is given by

$$
\begin{gathered}
M_{1}^{t}=-\int \sigma^{t} y d A=-\rho_{1}^{t} \varepsilon^{-t} P+\frac{b h}{J} \rho_{1}^{t} \sum_{j=1}^{J} \sigma_{1 j}^{t} \varepsilon_{1 j}^{t} ; \\
1=1, \ldots, I+1
\end{gathered}
$$

where equation (11) was used. Furthermore, Fig. 2(b) shows that the moment at the $1^{\text {th }}$ plane may be related to the eccentricity $\delta$ by the equation

$$
M_{i}^{t}=P\left\{\delta+\left(d_{s}^{t}-\frac{h}{2}\right)+u_{i}^{t}-L \frac{d u^{t} I+1}{d x}\right\}
$$

in which $d_{s}$ is the function $d(x)$ evaluated at the grip end, $u$ is the deflection, $L$ is the length of the pull rod, and

$$
d_{S}^{t}=h \frac{\varepsilon_{I B}^{t}-\varepsilon^{t}}{\varepsilon_{I B}^{t}-\varepsilon_{I T}^{t}}
$$

Equations (18) and (19) combine to yield

$$
\begin{aligned}
P\{\delta & \left.+\left(d_{s}^{t}-\frac{h}{2}\right)+u_{i}^{t}-L \frac{d u_{I+1}^{t}}{d x}\right\} \\
& =-\rho_{1}^{t} \varepsilon^{-t} P+\frac{b h}{J} \rho_{i}^{t} \sum_{j=1}^{J} \sigma_{1 j}^{t} \varepsilon_{1 j}^{t} ; i=1, \ldots, I+1
\end{aligned}
$$


In addition, the curvature relation is

$$
\left(\varepsilon_{1 B}^{t}-\varepsilon_{1 T}^{t}\right) / h=\left(u_{1+1}^{t}-2 u_{1}^{t}+u_{1-1}^{t}\right) / \Delta x^{2} ; 1-1, \ldots, I-1
$$

It 18 then easily shown that the following equations may be obtained

$$
\begin{aligned}
& \dot{\theta}_{1 j}^{t}=\left(\theta_{i j}^{t}-\theta_{1 j}^{t-\Delta t}\right) / \Delta t \\
& \theta_{i j}^{t}=\varepsilon_{i j}^{t}-\sigma_{i j}^{t} / E \\
& \varepsilon_{i j}^{t}=\varepsilon_{i T}^{t}+\left(\varepsilon_{i B}^{t}-\varepsilon_{i T}^{t}\right) \frac{21-1}{J} \\
& k_{c i j}^{t}=1-\beta_{c 1 j} \ln \left(\frac{\dot{\theta}_{1 j}^{t}}{\dot{\theta}_{R}}\right) \\
& z_{i j}^{t}=z_{i j}^{t-\Delta t}+k_{c i j}^{t}\left(\theta_{i j}^{t}-\theta_{i j}^{t-\Delta t}\right)
\end{aligned}
$$

where E 1s Young's modulus.

On the other hand, based on the discrete model, the creep constitutive equation should be used to correlate the stress and strain for the element $(1, j)$. For this element, equations (1), (2) and (10) are written as

$$
\begin{aligned}
\hat{k}_{c i j}^{t}= & \sigma_{y}^{0}\left(1+B \hat{z}_{1 j}^{t}\right) /\left\{\sigma_{i j}^{*}-\hat{k}_{1 j}^{t}-\frac{\sigma_{0}^{0}-\sigma_{y}^{0}}{\hat{k}_{i j}^{0}}\left(1+\hat{z}_{1 j}^{t}\right) .\right. \\
& {\left.\left[\left(\frac{1+B \hat{z}_{01 j}^{t}}{1+\beta_{i j}^{t}}\right)^{n}-\left(1+B \hat{z}_{i j}^{t}\right)^{-n}\right]\right\} }
\end{aligned}
$$




$$
\hat{z}_{1 j}^{t}=\hat{z}_{1 j}^{t-\Delta t}+\hat{k}_{c 1 j}^{t}\left(\hat{\theta}_{1 j}^{t}-\hat{\theta}_{1 j}^{t-\Delta t}\right)
$$

and

$$
\dot{\dot{\theta}}_{1\}}^{t}=\dot{\theta}_{R} \exp \left\{\left[1-\left[_{c 1 j}^{t}\right] / \hat{B}_{c 1\}}\right\}\right.
$$

so that

$$
\hat{F}_{1 f}^{t}=\sum_{t=0}^{t} \hat{\theta}_{1 j}^{t} \Delta t
$$

Note that the "hatted" quantities are those related to constitutive creep equations. These quantities should be matched by fteration with the "unhatted" quantities which appeared in the field equations.

fin assumption is made in arriving at these equations. It is assumed that the creep strain at a generic point in the specimen may be calculated by maintaining the same stress at this point throughout the creep stage. This, in effect, neglects the effirt of creep recovery and stress relaxation at fibers other than the N.A..

The matching of the above mentioned quantit_as: are represented by the equation

$$
\varepsilon_{1 j}^{t}=\hat{\varepsilon}_{1 f}^{t} ; \quad 1=1, \ldots, I+1 \text { and } f=1, \ldots, J
$$

This equation also represents the input by the constitutive creep equation into the formulation of the misalignment problem. Hence, the set of nonlinear equations $(17),(20),(21),(22),(27)$, and (32) represents the basic equations of the misalignment analysis. The unknown variables are $\varepsilon_{11,}^{t}, \varepsilon_{1 B}^{t}$, 
$\sigma_{1 f}^{t}, d_{s}^{t}, u_{1}^{t}$ and $z_{1 f}^{t}$ and, in the computation, these equations should be supplemented by equations (12), (13), (23), (24), (25) and (26). In solving

the set of nonlinear equations, the right hand side of equation (32) is consitered known.

The sei of nonlinear equations may be solved with the following boundary conditicns:

$$
\frac{d u^{t}}{d x}=0 \text { at } x=0
$$

and

$$
u^{t}=0 \text { at } x=\ell / 2
$$

provided that the inftial values (at $t=0$ ) of all the variables which correspond to a particular value of $\bar{\theta}_{0}$ are supplied from the results of Ref. [7].

An algorithm for the numerical solution of the above set of equations is presented in the Appendix. The theoretical results are discussed in the following section. 


\section{RESULTS AND DISCUSSION}

The sheet type rectangular specimens are used in this calculation. The geometrical data for the specimens are

$$
\begin{aligned}
& \text { specimen length }(l)=63.5 \mathrm{~mm} \\
& \text { specimen width }(h)=12.7 \mathrm{~mm} \\
& \text { specimen thickness }(b)=3.2 \mathrm{~mm} \\
& \text { eccentricity }(\delta)=0.05 \mathrm{~h}
\end{aligned}
$$

The influence of the pull rod length (L) is investigated by assigning two different ratios of $\mathrm{L} / \ell, \mathrm{i} . \mathrm{e} ., \mathrm{L} / \ell=2.0$ and 1.25 .

The influence of the preloading history on misalignment is investigated through the following consideration. The specimen is first subjected to a loading process of constant strain rate $\dot{\theta}_{0}=\dot{\theta}_{R}=1.30 \times 10^{-5} \mathrm{~s}^{-1}$ at the $\mathrm{N}$.A. The loading continues until a prescribed plastic strain $\bar{\theta}_{0}$ is reached at the N.A. The load is then kept constant and creep is subsequently computed. By considering different values of $\bar{\theta}_{0}$, the influence of prehistory may be studied.

In this calculation, three cases are considered, which are $\mathrm{L} / \mathrm{l}=2$, $\bar{\theta}_{0}=0.3 \% ; L / \ell=2, \bar{J}_{0}=0.5 \%$; and $L / \ell=1.25, \bar{\theta}_{0}=0.5 \%$. Results related to the finfluence of the pull rod length and the prehistory will be discussed later in the text. The initial data at the preloading stage, such as the Initial values of misalignment errors and the location of the N.A. prior to the creep stage are supplied by the misalignment program for the dynamic tension test reported in Ref. [7]. 
In order to reduce computer tIme, only four sections have been cut along both the longitudinal and the lateral directions of the specimen, 1.e., $I=4$ and $J-4$. Thus, the unknown varlables have been reduced to 55 in number and the set of nonlinear equations discussed in the previous section consists now of 55 equations. Although the number of elements in the discrete numerical model is at its mintmum, the model does provide conslatent numerical output under a variety of creep conditions. Since the area of interest for the misalignment analysis lies in the midspan of the specimin (the measuring devices are usually attached to the midspan), most of the results to be discussed are related to this area.

The material constants used in the calculation have been determined in Ref. [6]. They are

$$
\begin{aligned}
\sigma_{y}^{0} & =13.79 \mathrm{MPa}(2000 \mathrm{psi}) \\
\sigma^{\circ} & =27.92 \mathrm{MPa}(4050 \mathrm{psi}) \\
\mathrm{E}_{1} & =6.596 \times 10^{3} \mathrm{MPa}\left(9.566 \times 10^{5} \mathrm{psi}\right) \\
\mathrm{n} & =105 \\
B & =4.444
\end{aligned}
$$

The parameter $\beta_{c}$ of equation (3) has been found in Ref. [6] to depend on the creep stress. This dependence may be represented by the following inear equation (Fig. 4)

$$
\begin{aligned}
& \beta_{c i j}= 3.643 \times 10^{-2}-0.261 \times 10^{-1}\left(\sigma_{i f}^{*}-0.508\right) \\
& \quad \text { for } \sigma_{i j}^{*} \geq 0.493 \mathrm{MPa}
\end{aligned}
$$


where the creep stress is measured in MPa. For stress smaller than 0.493 MPa (or $3400 \mathrm{psi}$ ), the relationship between $\beta_{c}$ and $\sigma *$ is non-1inear. However, in all the cases considered in this calculation, the creep stress is in the range of equation (35) and the linear equation is used in the computation.

Figure 5 shows the normalized stress $\sigma_{i j}^{*} / \bar{\sigma}^{*}$ plotted versus time for the tup and bottom fibers in the three cases considered. It is seen that in all cases the variation in stress is minimal. The change in stress is less than $1 \%$ in the first hour of the creep test and is less than $2 \%$ in 8 hours. Therefore, the assumption made earlier that the effect of creep recovery and stress relaxation are negligible is a reasonable one. Figure 5 further shows that it is unlikely that a uniform stress distribution in the specimen can be reached before rupture.

It may also be observed from Fig. 5 that, for a fixed $\mathrm{L} / \mathrm{l}$ ratio, the deviation of the normalized stress from unity at any time $t$ is greater for smaller $\bar{\theta}_{0}$. The implication is that greater bending moment is obtained for smaller $\bar{\theta}_{0}$. This observation is related to the inherent error range for the extreme fibers at the pre-loading stage reported in Ref. [7]. A conclusion of Ref. [7] is that the error range for the extreme fibers is always decreasing with the increasing $\bar{\theta}_{0}$. Figure 5 also indicates that this error range in stress for a fixed $\bar{\theta}_{0}$ decreases with the creep time.

A second observation from Figure 5 is related to the length of the pull rod. The longer the pull rod is the smaller is the error range in stress. This observation is closely related to the magnitude of the end moment which will be further discussed. 
In Figure 6, the creep strains versus time for the top and bottom ftbers at the midspan of the specimen are shown and compared with the creep curver at the N.A. Although the N.A. shifts with time, it is convenfent :o 19 s: the creep response at the N.A. as a reference and compare those at othe fibers with it. It is seen from Fig. 6 that for a given $\bar{\theta}_{0}$, a shorter pull rod w1ll result in a greater variation in the magnitude of strain between the two extreme fibers. Furthermore, for a specific pull rod lengt 1 , the creep rate is smaller for smaller $\bar{\theta}_{0}$.

[.t appears in Fig. 6 that for all the three cases considered the creep rate of each fiber were almost identical to that of the N.A, and all creep curves, for each case were parallel to each other. A closer look at these curves will show that this is not true. Indeed this is made clearer by $\mathrm{Fi}_{\mathrm{i}}$ s. 7 and 8 , in which the differences in strains between the extreme fibers and between the bottom fiber and the N.A. are plotted against time t. These curves show two different trends of variation. For $\bar{\theta}_{0}=0.5 \%$, the differences ' i strains reach their peak values within one hour and decrease with time thereafter. On the other hand, in the case of $\bar{\theta}_{0}=0.3 \%$, the strain differences increase with the creep time up to 8 hours. Due to the 1 imjtation in computer funds, the computation is terminated at 8 hours creer time. But $i$ is conjectured that these curves, would reach their peaks at a late time and show a downward trend thereafter. Thus, the peaks wou." : occur early for a large $\bar{\theta}_{0}$.

F. gure 9 shows the difference in strains between the geometrical center11 i and the N.A. at the midspan. It is seen that these differences in strain are alw:ys decreasing with time for all the cases considered. However, 
the computation time is not long enough to see if the differences will eventually vanish with time. Nevertheless, it may be concluded, that for the same pull rod length, the smaller the prestrain $\bar{\theta}_{0}$ is the longer is the creep time required to close the difference between $\varepsilon_{c}$ (the strain at the centerline) and $\bar{\varepsilon}$ (the strain at the N.A.).

Perhaps the most significant of all. the results of this paper is tha: presented in Fig. 9. The implication of this result is that the difference between $\varepsilon_{c}$ and $\bar{\varepsilon}$ will decrease with time in spite of the test system misalignment. That is, the creep strain measured by an extensometer, which is also the strain at the geometric centerline $\varepsilon_{c}$, will become closer and closer to the creep strain of a perfectly aligned test system at large time. Therefore, it may be concluded that the effect of misalignment in a creep test is sibnificant only in the primary creep range. In a long time creep test, the effect of misalignment is less important, because the plastic deformation will even out the non-uniform distribution of strain with time. This finding is not in agreement with those of Penny and Leckie [1] and Hayhurst [4]. The analyses of Ref. [1] and [4] predicted that the eccentricty cf the test system would cause the creep curve to deviate increasingly with time from the creep curve of the perfectly aligned test system.

The error of misalignment at the geometric centerline (or center error) is defined by $E_{c}=\left(\varepsilon_{c}-\bar{\varepsilon}\right) / \bar{\varepsilon}$. The center errors at the midspan for the three cases under consideration are shown in Fig. 10. It is seen that the center error decreases with time for all cases. The errors are within $2 \%$ at the onset of creep and become less than $0.4 \%$ after 8 hours of creep. The same trend is found in the curves of Fig. 11 for the errors of top and bottom fibers. These errors are within $30 \%$ at the onset of creep and are about $8 \%$ to $12 \%$ after 8 hours of creep. 
The shifting of the N.A. and the end moment distribution are closely related by equation (19) and are plotted in Figs. 12 and 13 against time. All curves are of the decreasing type with time. However, a conclusion has not been reached concerning the questions of whether the N.A. would eventually coincide with the geometric centerline and whether the end moment would eventually be reduced to zero at large time. More computations are needed in order to answer the above questions.

Finally, the deflection curves of various creep times are plotted in Fig. 14. For the cases of $L / l=2, \bar{\theta}_{0}=0.5 \%$ and $L / l=1.25, \bar{\theta}_{0}=0.5 \%$, the deflection curves reach their maximum position within 2 hours and then decrease their magnitudes thereafter. For the case of $L / l=2$ and $\bar{\theta}_{0}=0.3 \%$, the magnitude of the deflection curve increases with time for the 8-hour. creep time period. It is conjectured that the deflection would reach its maximum position at a later time and the magnitude would then decrease with time. 


\section{CONCLUSIONS}

The formulation of a misaligned specimen subjected to the creep test has been presented. The Investigation is restricted to the symmetric case of test system misalignment. The specimens used in the calculation are the sheet type rectangular specimens of 1100-0 aluminum. Three cases have been investigated in detail. They are the case of $\mathrm{L} / \ell=2$ and $\bar{\theta}_{0}=0.3 \%$, the case of $L / \ell=2$ and $\bar{\theta}_{0}=0.5 \%$, and the case of $L / \ell=1.25$ and $\bar{\theta}_{0}=0.5 \%$. The following results have been obtained from this investigation:

(1) During the creep test, the stress is quite constant for all fibers. The total change in stress for the 8-hour creep period for the top and bottom fibers is within $2 \%$ for all cases considered.

(2) The difference between the creep strains at the geometric centerline and the N.A. decreases with the creep time in spite of the test system misalignment.

(3) The center error and the errors at the extreme fibers are always decreasing during creep.

(4) For the same pull rod length but with different amounts of prestrain, the smaller the prestrain is (in the plastic range) the greater is the misalignment error at the onset of creep and the longer creep time it takes for the misalignment effect to vanish. For the same prestrain but with different pull rod lengths, the error is greater for the shorter pull rod than for the longer.

(5) In order to effectively reduce the misalignment error in a creep test, long pull rod should be urad and the test should be conducted with a large prestrain. 


\section{vI. ACKNOWLEDGEMENT}

The authors are grateful to NASA-Langley Research Center, Hampton, Virginia for the support of this research under Grant No. NSG 1499. Dr.

D. R. Rummler is also thanked fcr providing the NONLIN subroutine which was instrumental in obtaining the numerical solutions reported herein. 


\section{REFERENCES}

1. Penny, R. K., and Leckle, F. A., "The Mechanics of Tensile Testing," International Journal of Mechanical Sclence, Vol, 10, 1968, Pp. 262-273.

2. Christ, B. W., and Swanson, S. R., "Alignment Problems in the Tensile Test," Journal of Testing and Evaluation, Vol. 4, 1976, pp. 405-417.

3. Wu, H. C. and Rummler, D. R., "Analysis of Misalignment in the Tension Test," ASME Journal of Engineering Materials and Technology, Vol, 101, 1979, pp. 68-74.

4. Hayhurst, D. R., "The Effects of Test Varlables on Scatter in HighTemperature Tensile Creep-Rupture Data", International Journal of Mechanical Science, Vo1. 16, 1974, pp. 829-841.

5. Penny, D. K., Elilison, E. G. and Webster, G. A., "Specimen Alignment and Strain Measurement in Axial Creep Tests", Materials Research and Standard, February 1966, pp. 76-84.

6. Wu, H. C. and Chen, L., "Endochronic Theory of Transient Creep and Creep Recovery," Report G302-79-001, Division of Materials Engineering, The University of Iowa, April 1979.

7. Wu, H. C., Wang, T. P. and Yip, M. C., "The Effect of Test Symstem Misalignment in the Dynamic Tension Test", Report G302-80-001, Division of Materials Engineering, The University of Jowa, March 1980. 


\section{APPENDIX}

\section{Algorithm for Numerical Solution of the Misalignment Problem in Creep}

In this calculation, the specimen is discretized into 4 elements along both the longitudinal $(I=4)$ and the lateral $(J=4)$ directions. Algebraically, there are 55 nonlinear equations with 55 unknown variables. They are:

Equations: eq. (17) -5 , eq. (20) -1 , eq. (21) -5 , eq. (22) -4 , eq. (27) -20 , eq. (32) -20 .

Variables: $\sigma_{1 j}^{t}-20, \varepsilon_{1 T}^{t}-5, \varepsilon_{1 B}^{t}-5, z_{1 j}^{t}-20, u_{1}^{t}-4, d_{s}^{t}-1$.

The 55 variables are denoted by $X(55)$. The computation is designed to achieve the following three tasks: (a) To compute the strain $\hat{\varepsilon}_{1 j}^{t}$ in the element $(i, j)$ from the constitutive creep equations. (b) To match the strain $\hat{\varepsilon}_{i f}^{t}$ with the strain $\varepsilon_{i j}^{t}$ and solve the 55 nonlinear equations. (c) To iterate the step (b) until $\varepsilon_{i j}^{t}=\hat{\varepsilon}_{i j}^{t}$ and $\sigma_{i j}^{t}=\sigma_{i j}^{*}$.

Step (a) Includes setting up m (approximately .00) creep stress levels between the extremum stresses in the specimen. These are generally the stresses at the top and bottom fibers of the grip end. The m stress levels should include the creep stress $\bar{\sigma}^{*}$ at the N.A. The initial values $(a t=0)$ $z_{\mathrm{m}}^{0}, \mathrm{k}_{\mathrm{m}}^{0}, \varepsilon_{\mathrm{m}}^{0}$ for each stress level are found by iteration. The $\mathrm{m}$ creep strains for each time step are then found from equations (28), (29), (30), (31) and (35) with the subscripts if replaced by $m$ in these equations.

In Step (b), the initial guess of the variables is modified by

$$
x^{t}=x^{t-\Delta t}\left(\bar{z}^{t} / \bar{z}^{t-\Delta t}\right)
$$


The 20 stresses $X(1) \sim X(20)$ are then matched with the m stress levels found in Step (a). Linear interpolation may be used between stress levels. Thus, the creep strains $\hat{\varepsilon}_{1 f}^{t}$ may be obtained.

Putting the values of $\hat{\varepsilon}_{1 f}^{t}$ found in Step (b) into the right hand side of equation (32), the set of 55 equations is then solved numerically. However, the stresses $c_{1 j}^{t}$ found may not agree with the 20 stress levels which give rise to $\hat{\varepsilon}_{1 f}^{t}$. Thus, in Step (c) an iteration procedure is employed to achleve the goal that $\sigma_{1 j}^{t}=\sigma_{1 j}^{*}$ when $\varepsilon_{i f}^{t}=\widehat{\varepsilon}_{1 j}^{t}$.

The above procedure is repeated for $t+\Delta t$. 


\section{FIGURE CAPTIONS}

F1g. 1 Strain History of a Creep Test

Fig. 2 (a) Specimen Dimensions and Coordinate System

(b) Load Train Configuration of the Symmetric Case of

Misalignment

Fig. 3 The Discrete Numerical Model

Fig. 4 Stress Effect on $\beta_{c}$

Fig. 5 Stress Varlation During Creep

F1g. 6 Creep Strains for Misaligned Specimens

Fig. 7 Strain Difference Between Extreme Fibers

Fig. 8 Strain Difference Between Bottom Fiber and N.A.

Fig. 9 Strain Difference Between Geometric Centerline and N,A.

F18. 10 Center Error vs. Creep Time

F1g. 11 Errors at Top and Bottom Fibers

Fig. 12 Shifting of N.A.

Fig. 13 End Moment

Fig. 14 Deflection Curves 


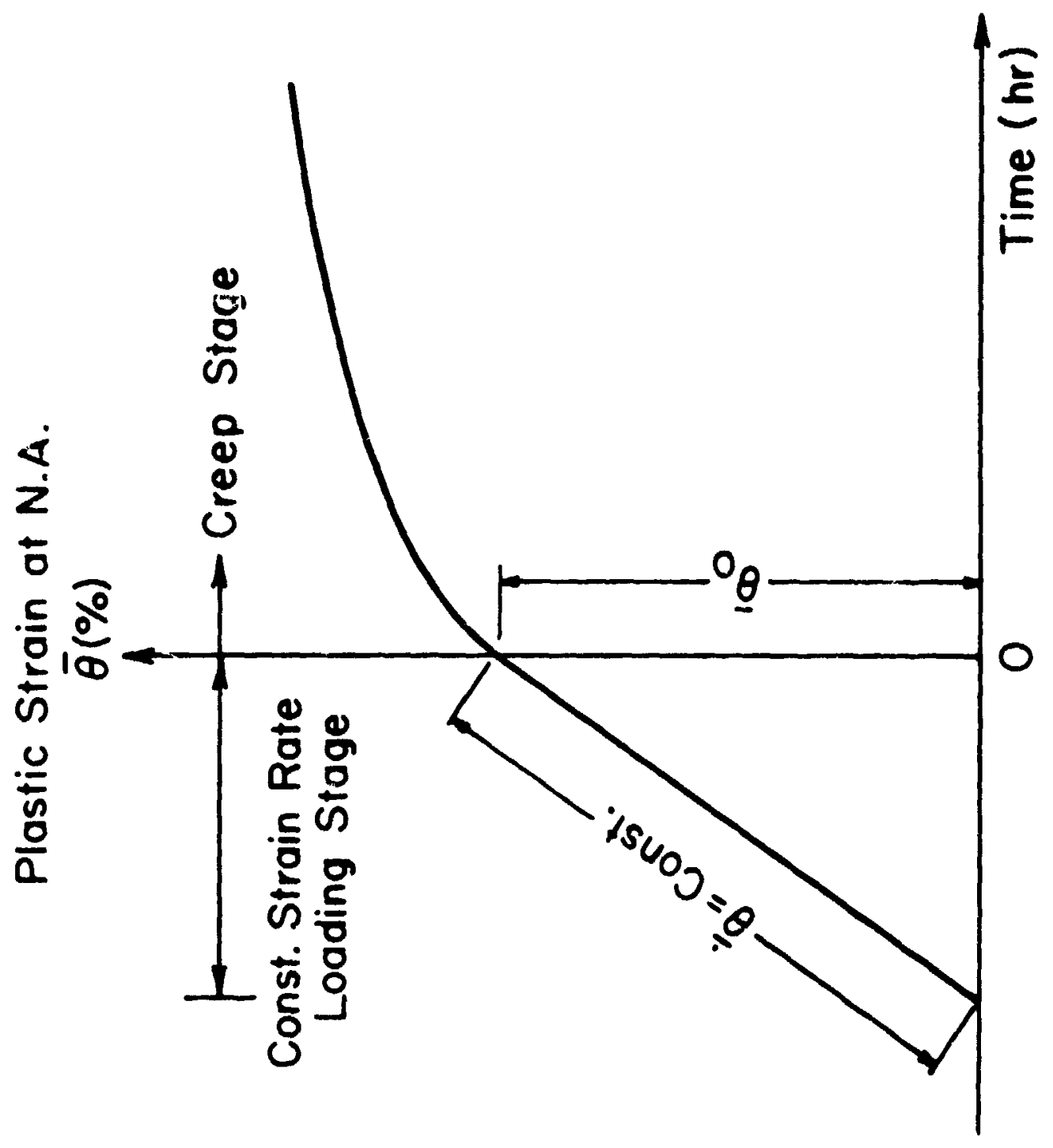


(a)
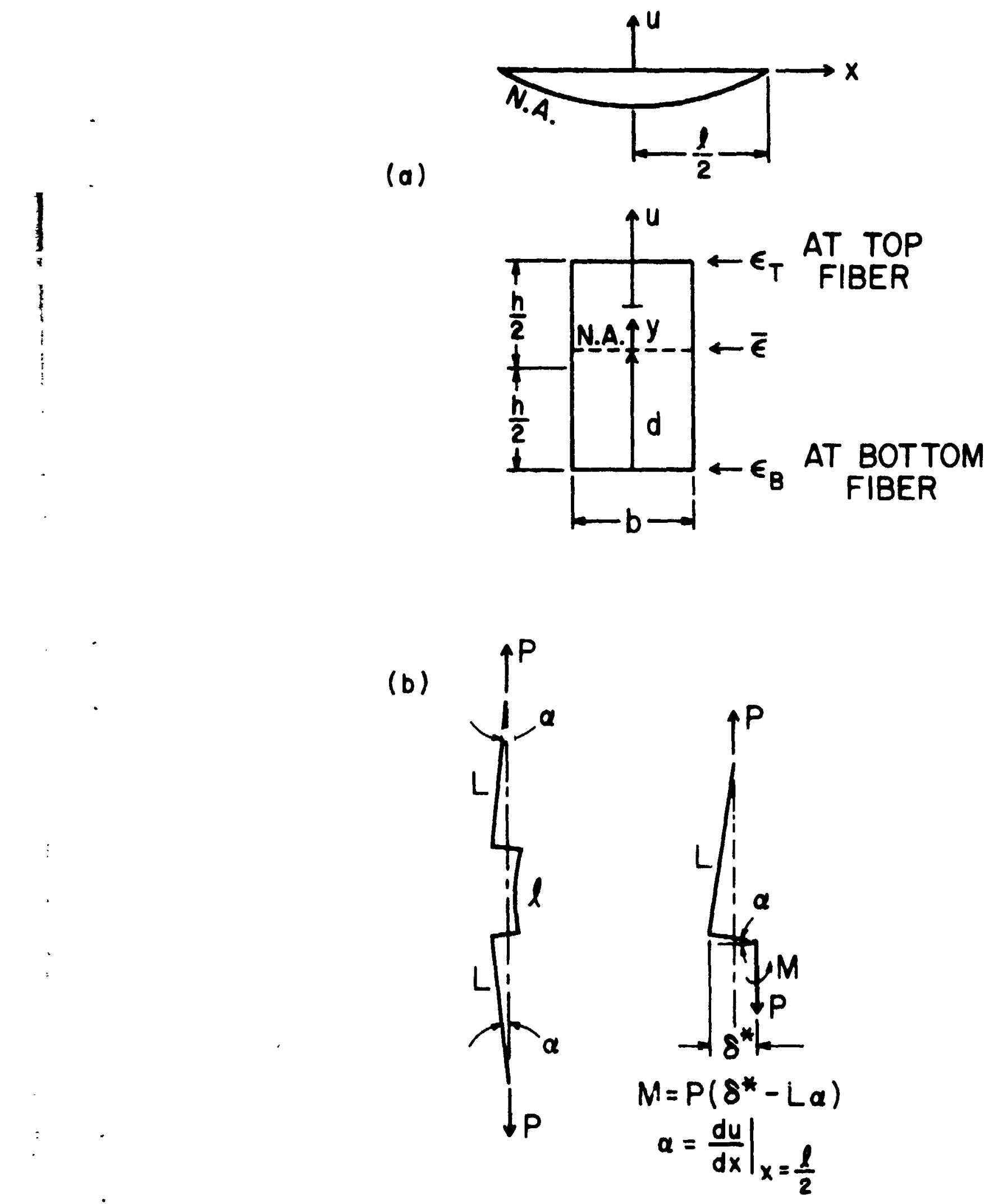

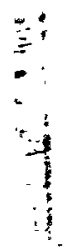




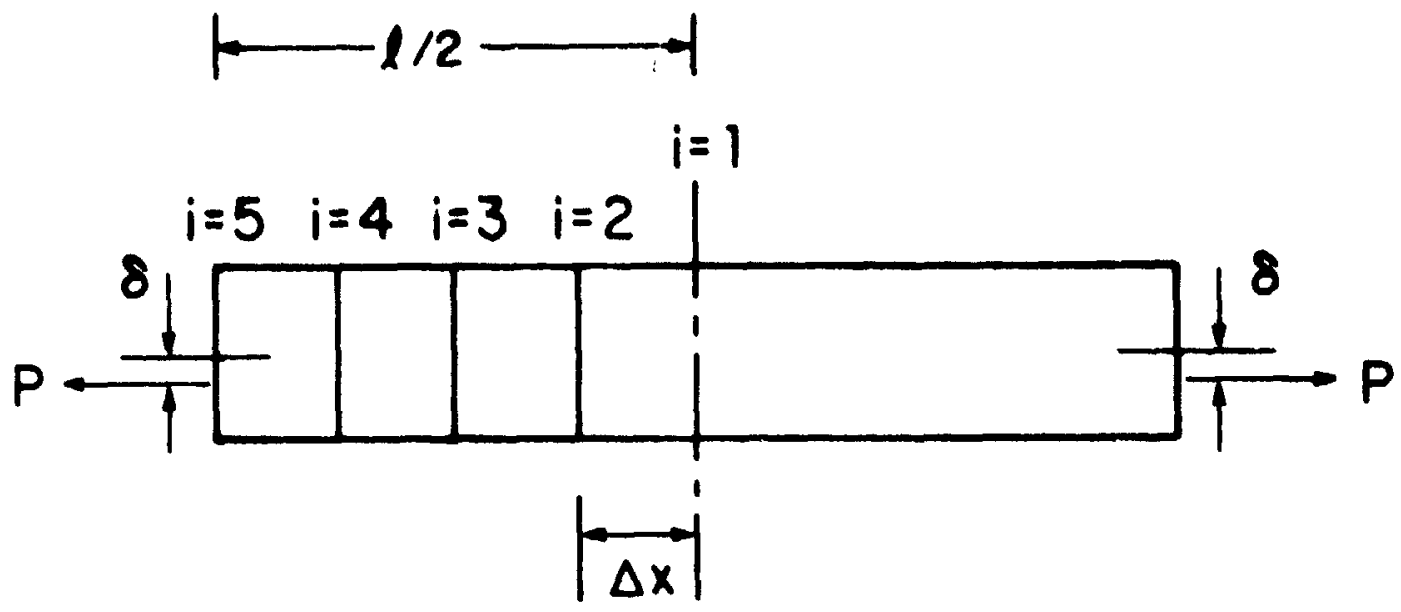

(a)
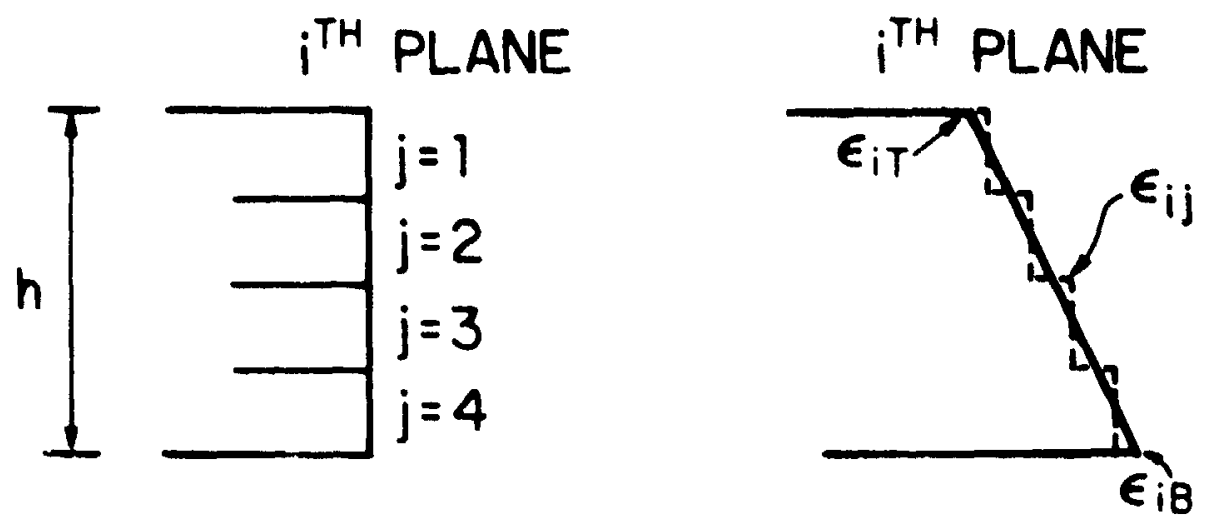

(b)

---DISCRETIZED STRAIN

- Actual STRAIN IN THE $i^{\text {TH }}$ PLANE 
(DdW)

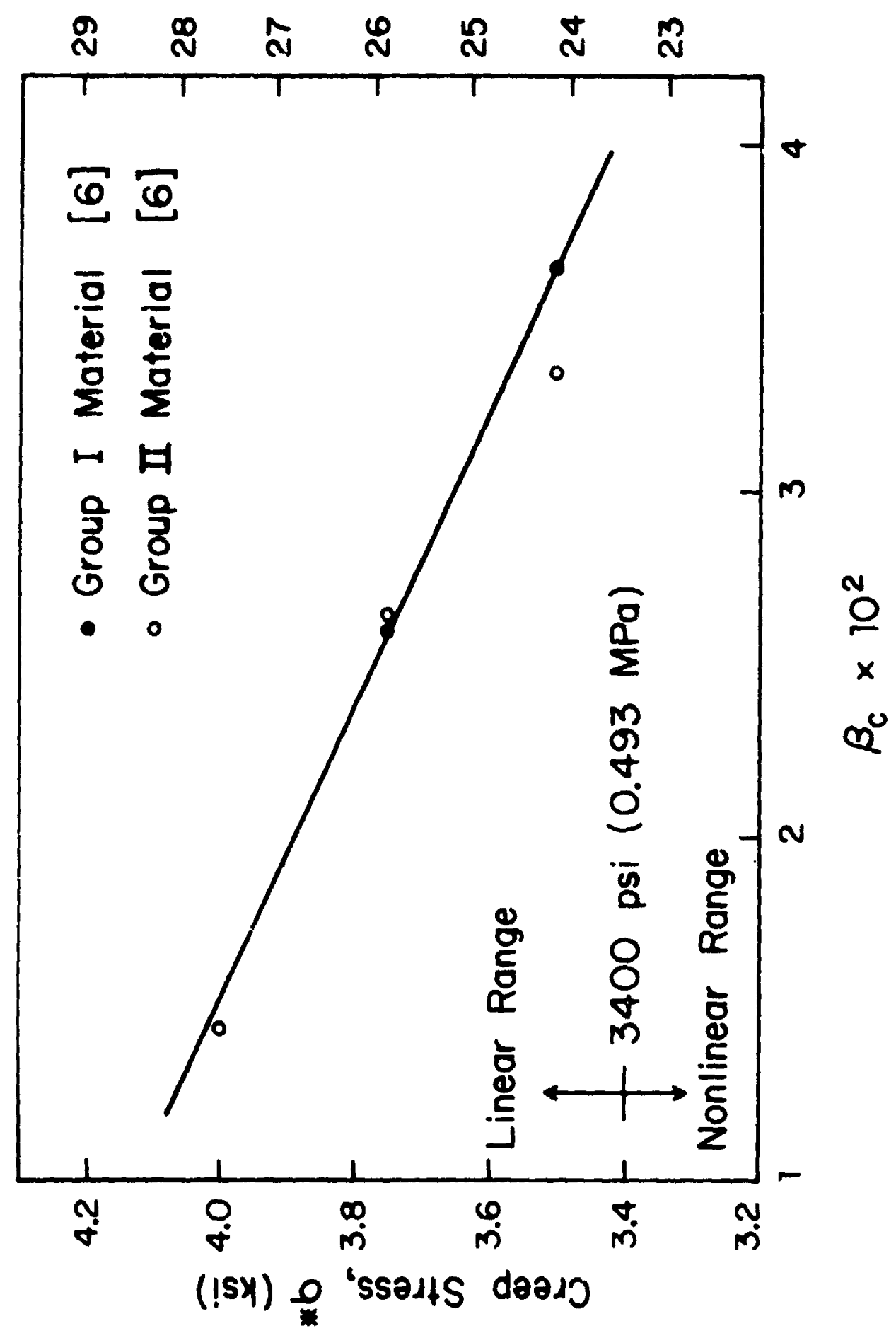



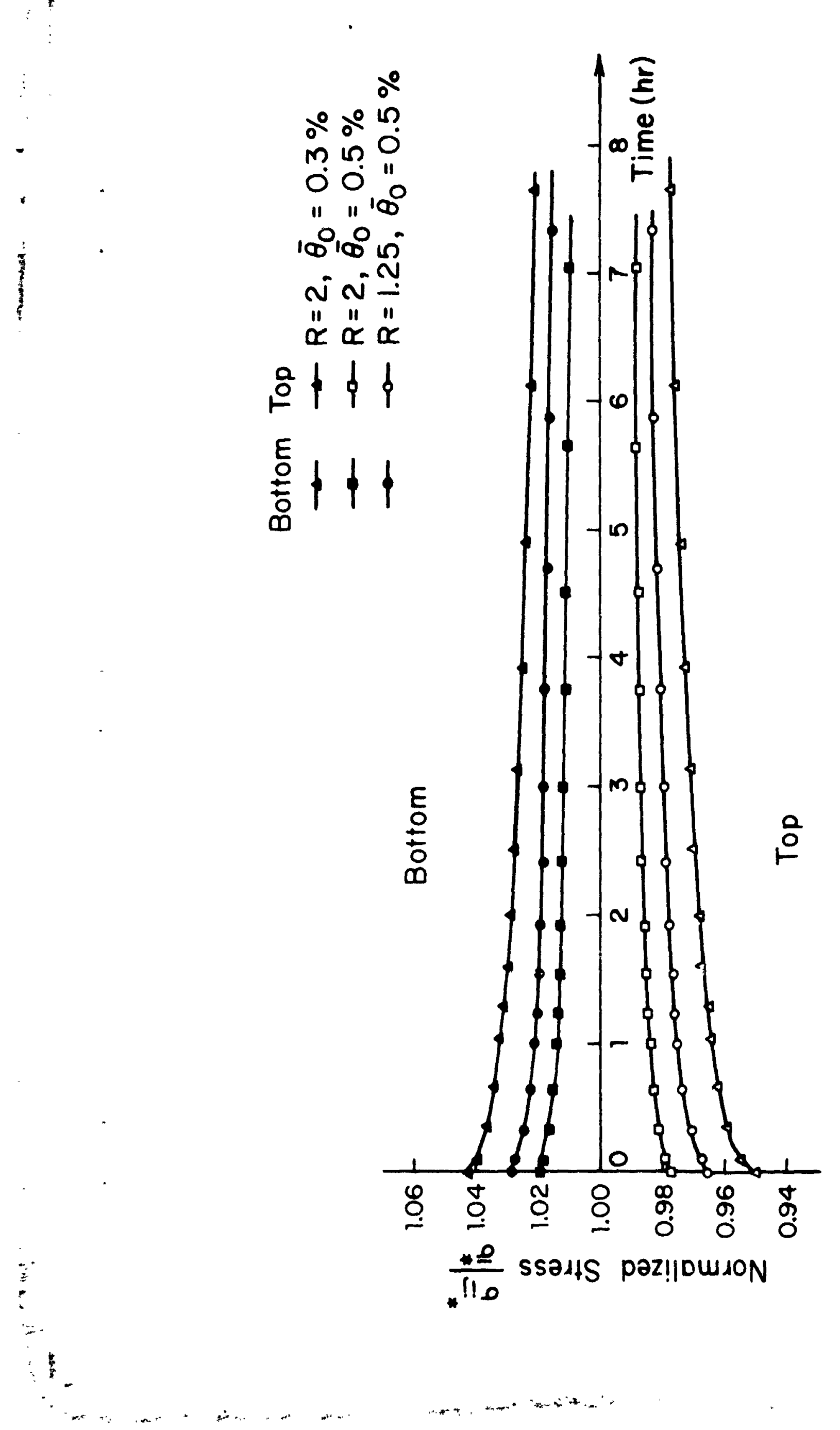


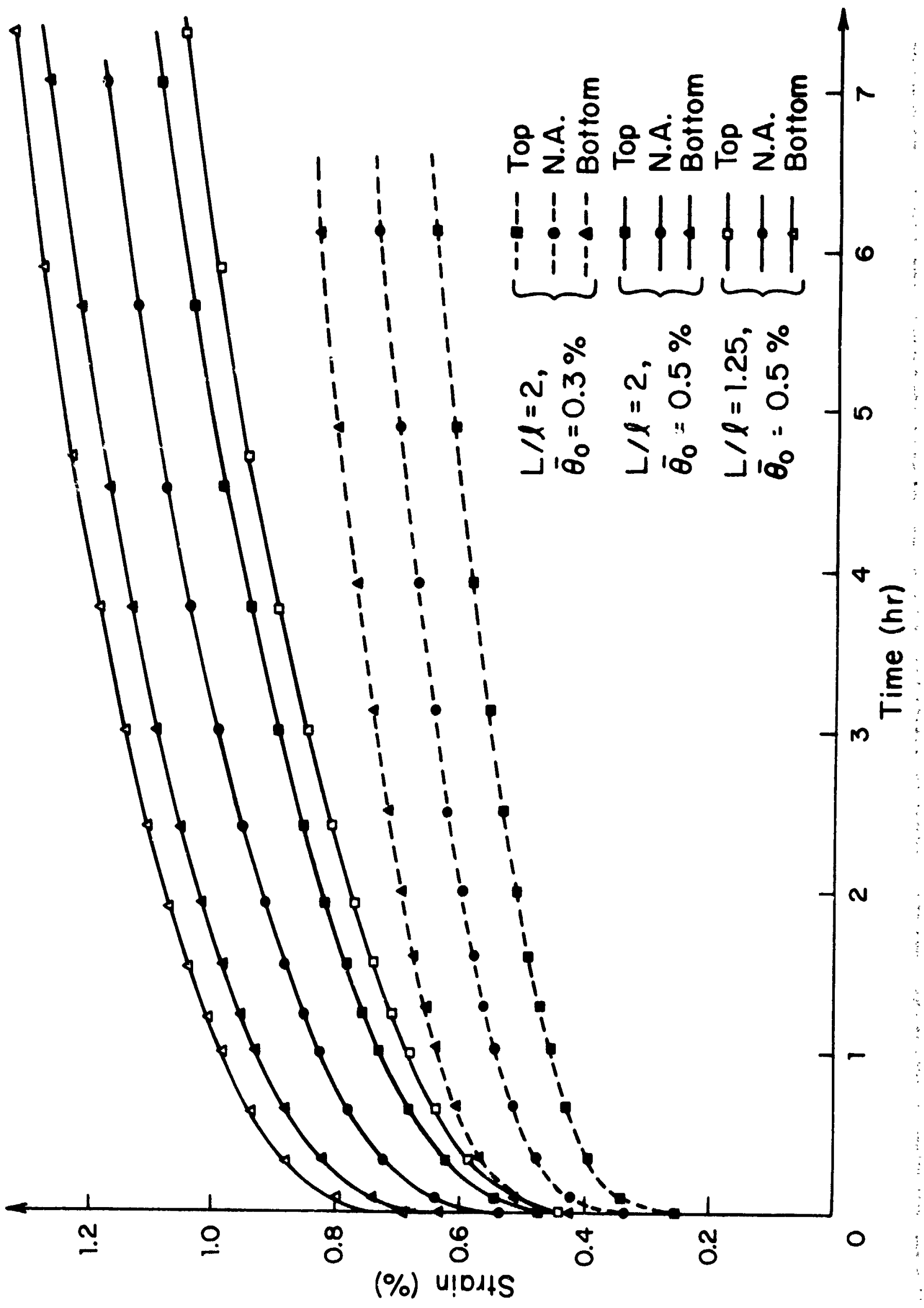




$$
4
$$




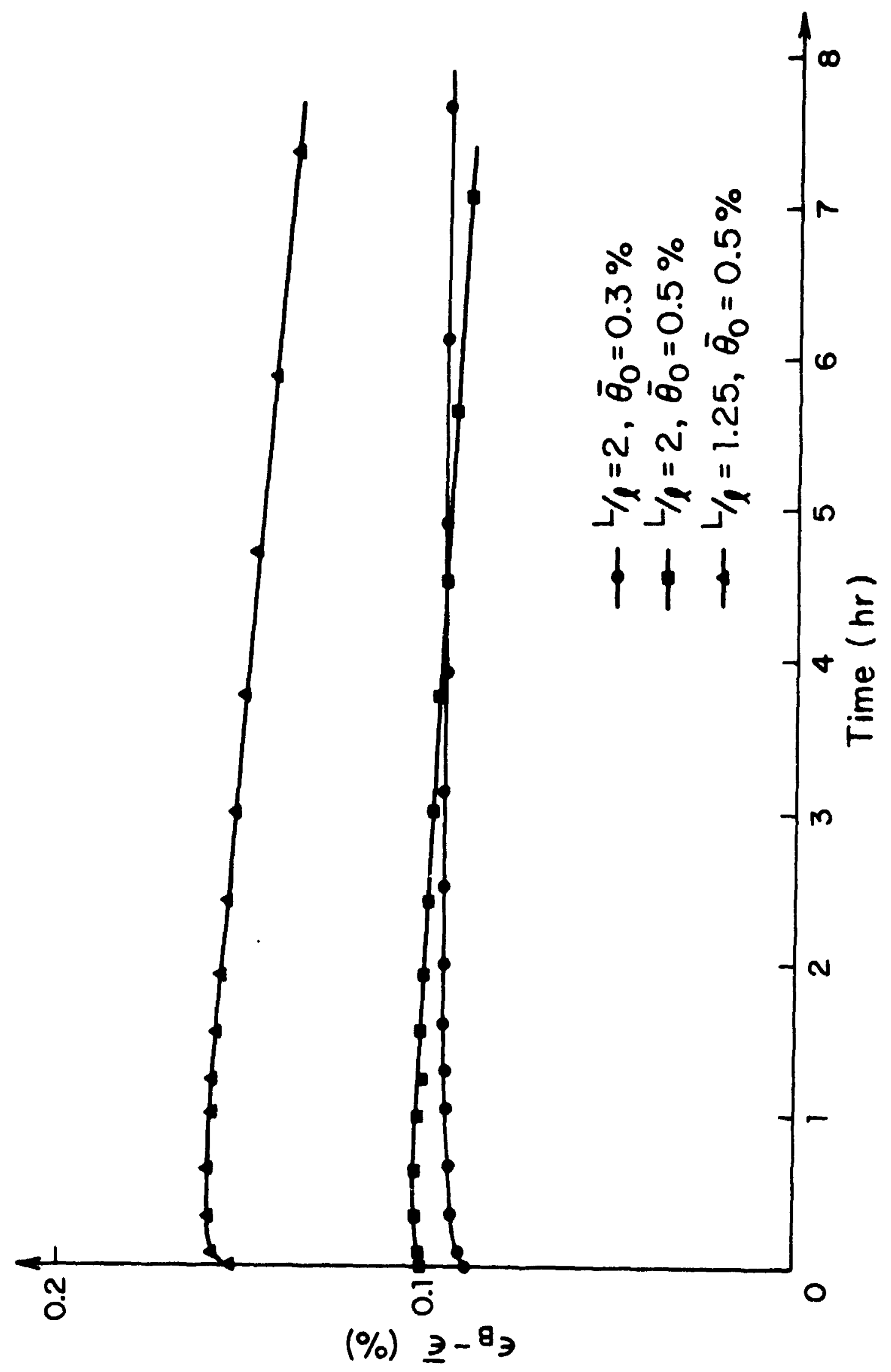




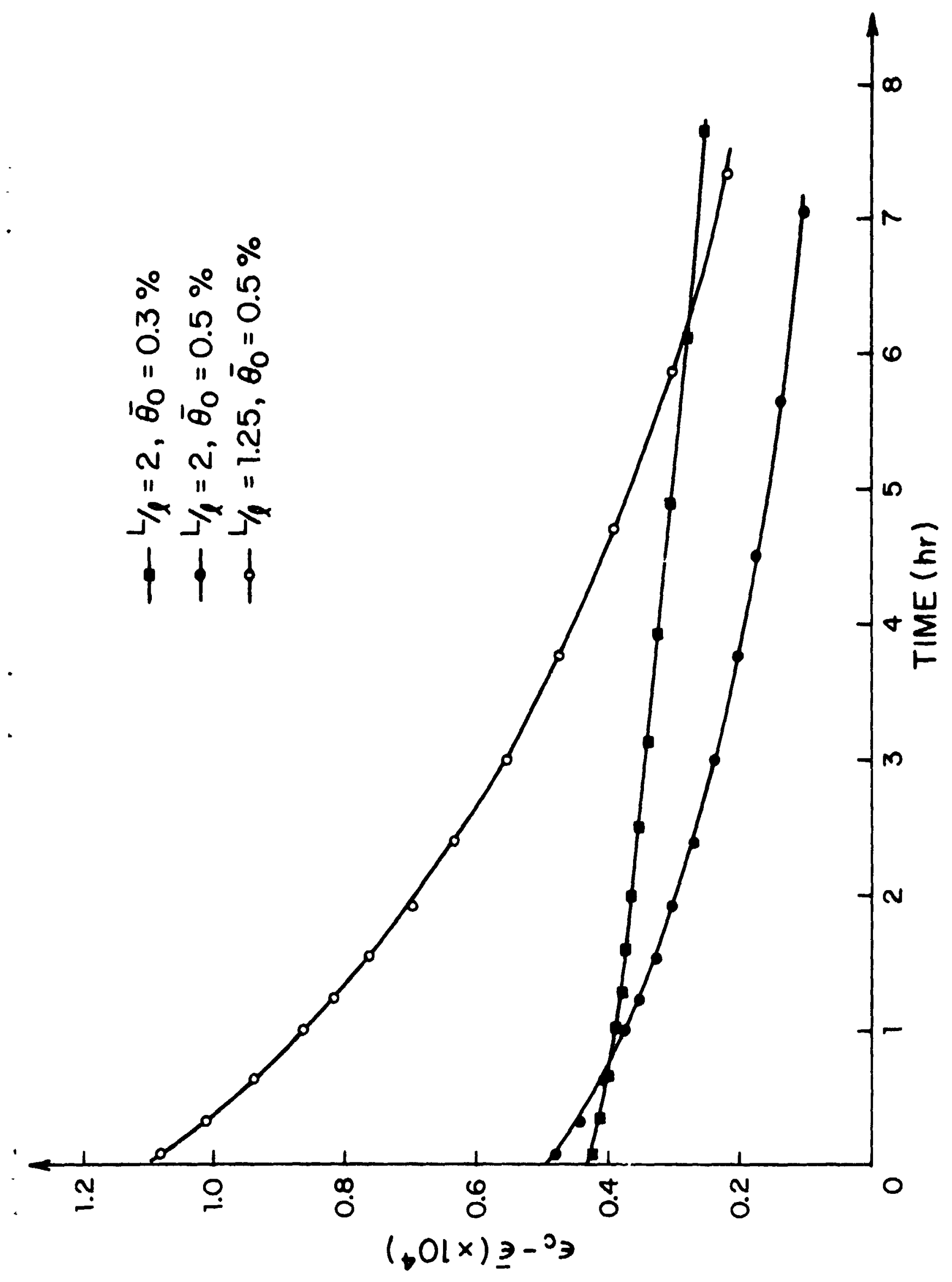




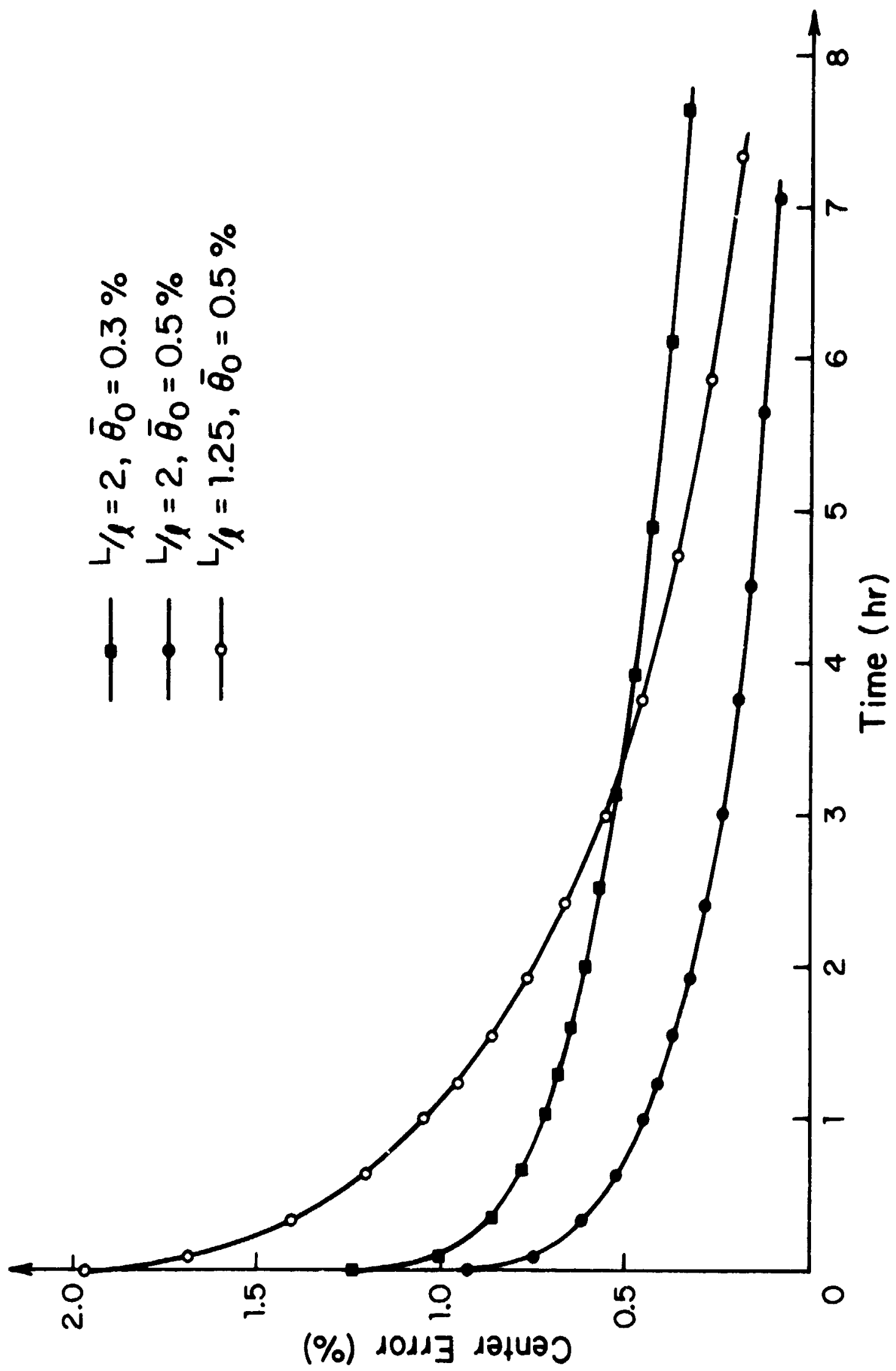




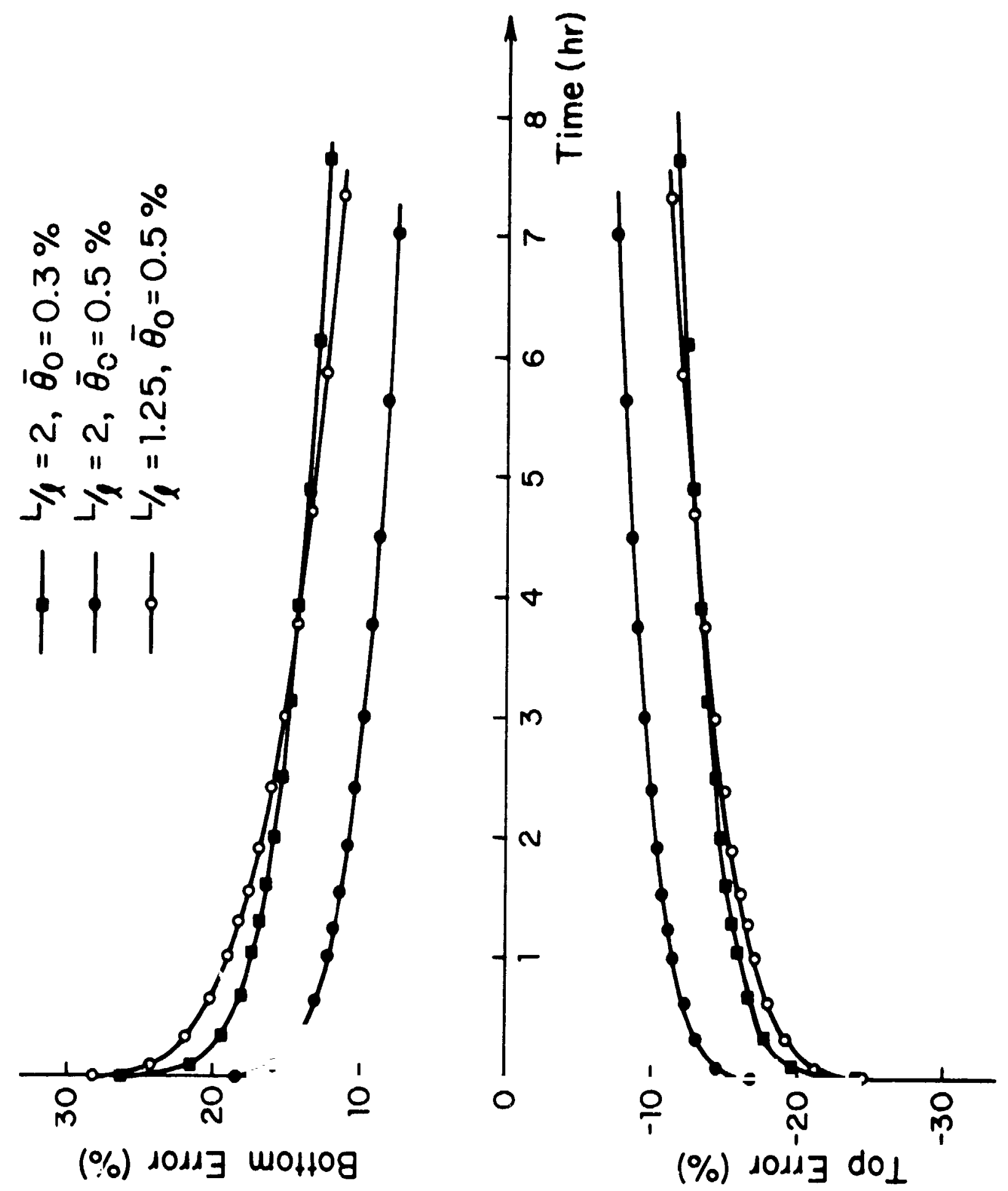




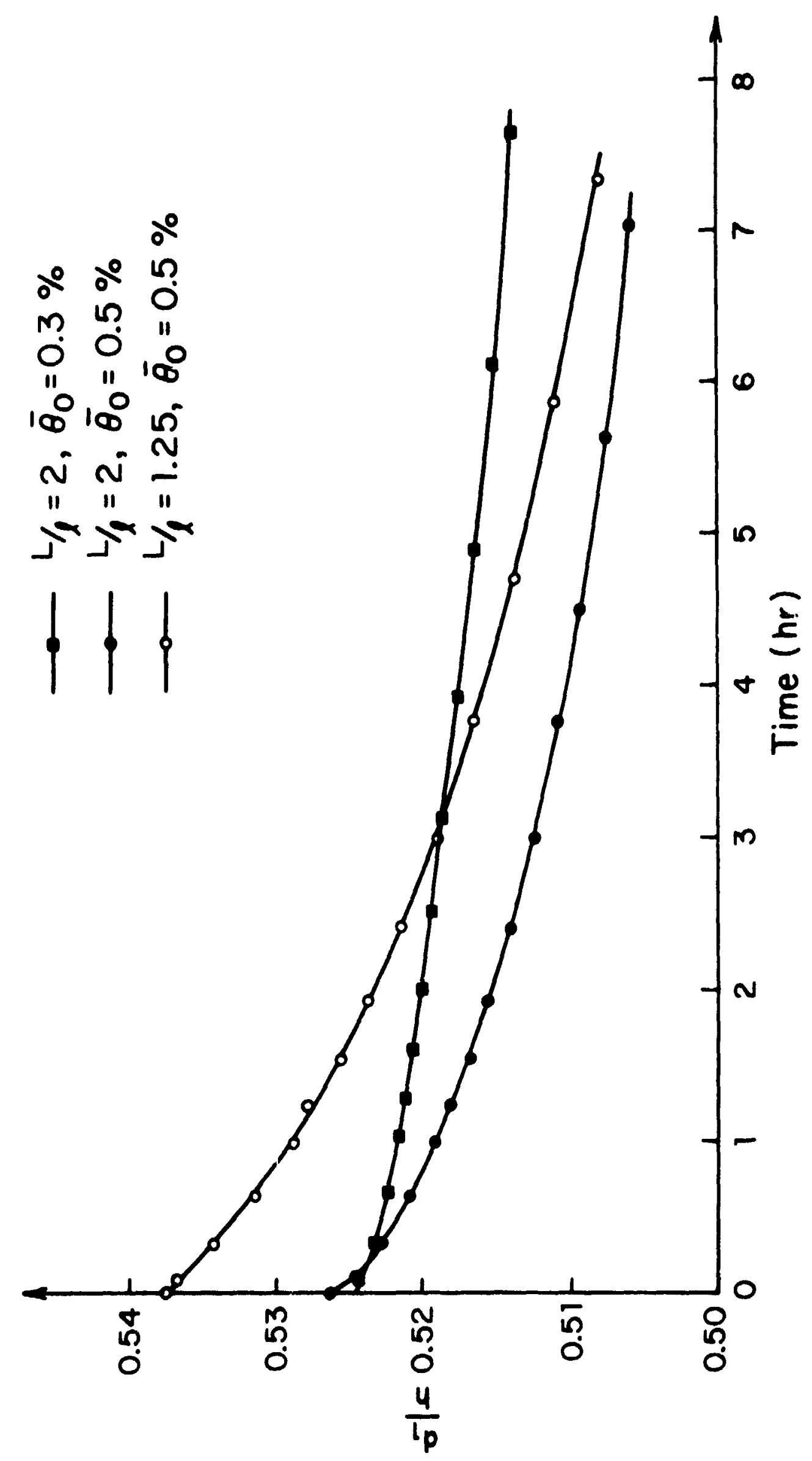




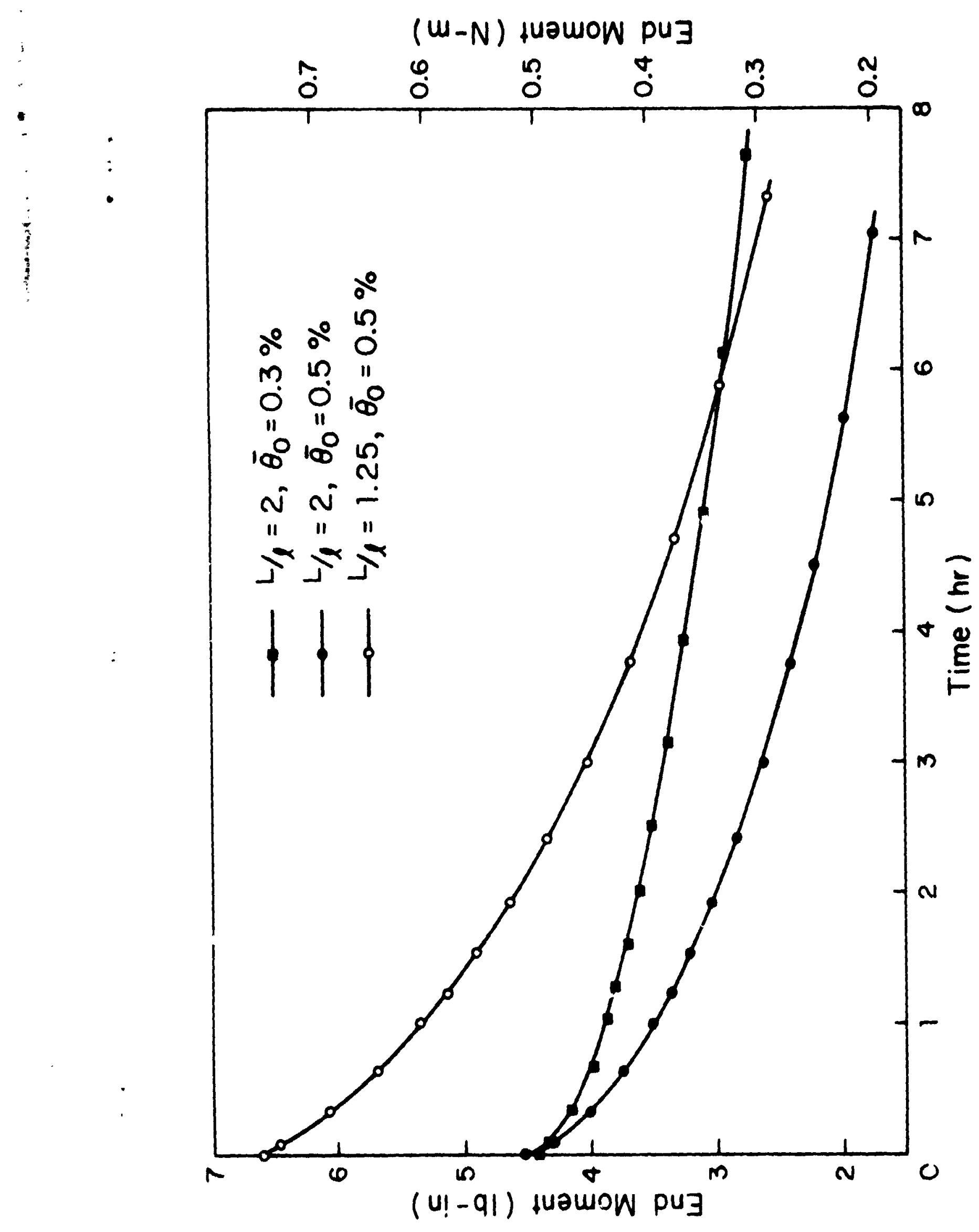




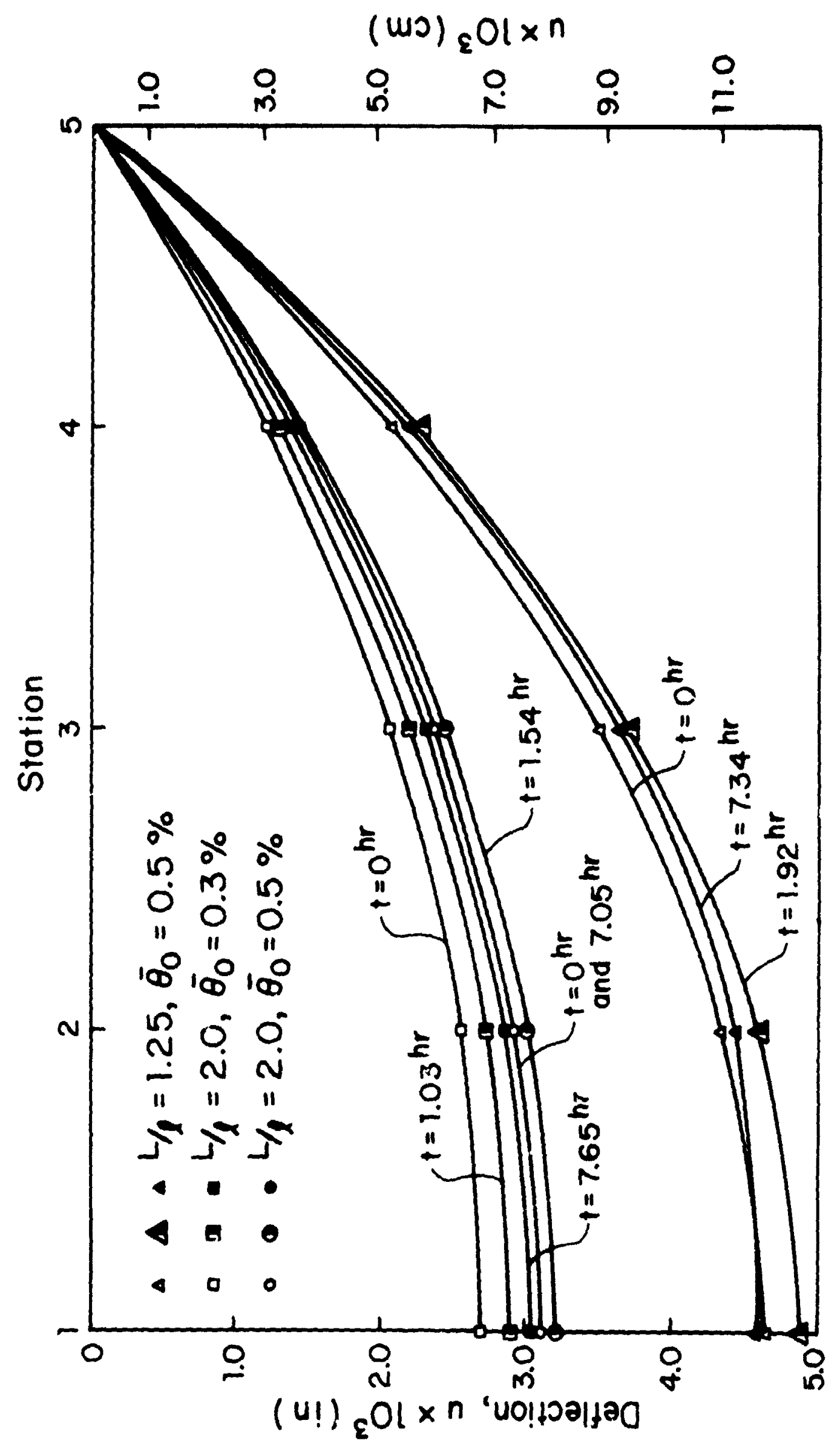

